\title{
Design and Assembling of a Magneto-Inertial Wearable Device for Ecological Behavioral Analysis of Infants
}

\author{
F. Taffoni, D. Campolo, J.Delafield-Butt, F. Keller, E. Guglielmelli.
}

\begin{abstract}
There are recent evidence which show how brain development is strictly linked to the action. Movements shape and are, in turn, shaped by cortical and sub-cortical areas. In particular spontaneous movements of newborn infants matter for developing the capability of generating voluntary skill movements.

Therefore studying spontaneous infants' movements can be useful to understand the main developmental milestones achieved by humans from birth onward.

This work focuses on the design and development of a mechatronic wearable device for ecological movement analysis called WAMS (Wrist and Ankle Movement Sensor). The design and assembling of the device is presented, as well as the communication protocol and the synchronization with other marker-based optical movement analysis systems.
\end{abstract}

\section{INTRODUCTION}

$\mathrm{S}^{\mathrm{s}}$ TUDYING child development could be a source of a great amount of data useful to understand human behaviours, neuro-biological mechanisms of brain development and main developmental milestones achieved by humans from birth onwards.

Neuro-Developmental Engineering (NDE) is a new interdisciplinary research area at the intersection of developmental neuroscience and bioengineering, aiming at providing new technologies and methods to study exactly these topics.

There are recent evidence which show how brain development is strictly linked to the action [1]: movements shape and are, in turn, shaped by cortical and sub-cortical areas. Understanding the meaning of an action or how to use an instrument seems to be possible thanks to the capability to replay that action or the use of that specific instrument. In particular the development of tool use ability seems to be a continuous process during which infants show a trial and error behavior which can be considered as self-generated opportunities for perceptual learning [2]. Alterations in this process could be a sign of a modified neurological

This work was supported by a grant from the European Union, FP6NEST/Adventure Programme, contract no. 015636, and by a grant from "Regione Lazio" under the "DOCUP 2000/2006 - Sottomisura II.5.2 progetto ITINERIS” Programme.

F. Taffoni, D.Campolo, E. Guglielmelli are with Biomedical Robotics and Biomicrosystem Lab, Università Campus Bio-Medico, Rome, Italy (email: \{f.taffoni, d.campolo, e.guglielmelli\}@unicampus.it).

J.Delafield-Butt is with Perception in Action Laboratory, University of Edinburgh, Edinburgh, UK (e-mail:jtdb@holyrood.ed.ac.uk>)

F.Keller is with Developmental Neuroscience Laboratory, Università Campus Bio-medico, Rome, Italy (e-mail f.keller@unicampus.it) development. Therefore one of the reasons for studying spontaneous movements in young infants could be a diagnostic one, that is, giving important supplementary information to the traditional test used [3][4][5][6].

Infants' motor behavior using marker-based optical movement analysis systems has been studied in several works [3][7][8]. These systems usually require a structured environment: typically infants are seated in a chair reclined at some degrees with respect to a vertical axis and surrounded by cameras, that is, infants are not in their natural environment. Moreover limbs movements are subject to line-of-sight issues when marker-based optical systems are deployed. To solve this problem multiple cameras have to be used making the system expensive and hardly usable outside a motion analysis laboratory (i.e. not ecologic).

Spontaneous movements of full-term newborn infants have been studied by Ohgi et al. [9] using triaxial accelerometerbased sensors. Authors found that the production of infants' spontaneous movements involves chaotic dynamic systems that are capable of generating voluntary skill movements. In particular, nonlinear time series analysis, applied to data relative to infants' spontaneous movements, suggested that they are characterized by a nonlinear chaotic dynamics with 5 or 6 embedding dimensions.

Although triaxial accelerometers are available in highly miniaturized cases, they are not sufficient to reconstruct the spatial features of movement in 3D [10]. In particular orientation can be measured very well by means of the magneto-inertial technology. Embedding magneto-inertial sensors in normal toys could be useful to study manipulation task in very young children during first months of life [11]. The main drawback of such a solution is that child must handle toy to have data on his or her movement.

The aim of our research is assessing the full spatial features of all infants' spontaneous movements in 3D by deploying a complete, wearable Inertial Navigation Systems (INS) [10].

This paper focuses on the design of a wearable device for movement analysis in infants as young as two weeks of age called Wrist \& Ankle Movement Sensors (WAMS). This device has been designed to solve and improve respectively occlusion and portability issues detailed above. It has been devised to be used as a stand-alone solution for movement analysis as well as a part of a pre-existing setup by means of synchronization. 


\section{FUNCTIONAL AND TECHNICAL SPECIFICATIONS}

WAMS devices must not modify natural infant's movement such as hand flapping, foot flapping, reaching to grasp movements, etc. Such a consideration leads to small size/weight requirements in order to guarantee an easy and comfortable wearing of the device by very young infants ( from 2 weeks up).

\section{A. Mechanical Constraints}

According to anthropometric data (see [12] and tables below) the device size should not exceed $3 \mathrm{~cm}$ in width and about 4-5 cm in length .

TABLE I

WRIST CIRCUMFERENCE [cm]

\begin{tabular}{llllllll}
\hline Age $(\mathrm{mos})$ & $\mathrm{N}$ & Mean & s.d & Min & $5^{\text {th }}$ & $50^{\text {th }}$ & $95^{\text {th }}$ \\
\hline $0-2$ & 31 & 9.1 & 0.9 & 7.8 & 7.9 & 8.8 & 10.6 \\
$3-5$ & 44 & 10.2 & 0.9 & 8.3 & 8.5 & 10.2 & 11.1 \\
\hline
\end{tabular}

TABLE II

LOWER HARM LENGTH [cm]

\begin{tabular}{llllllll}
\multicolumn{7}{c}{ LOWER HARM LENGTH $[\mathrm{cm}]$} \\
\hline \hline Age $(\mathrm{mos})$ & $\mathrm{N}$ & Mean & s.d & Min & $5^{\text {th }}$ & $50^{\text {th }}$ & $95^{\text {th }}$ \\
\hline 3 & 139 & $\mathbf{1 4 . 8}$ & 1.1 & 13.1 & 14.8 & 14.8 & 10.6 \\
6 & 99 & 16.9 & 1.2 & 14.9 & 16.8 & 16.8 & 11.1
\end{tabular}

Its weight should not exceed $20 \mathrm{~g}$ at all [13]. It should be provided with an easy and variable system, like Velcro straps, to fix it to the anatomical infant's segments.

\section{B. Measuring Kinematics}

Particular attention was paid in the choice of the most suitable technologies for the sensing unit. Although motion tracking can count on a host of different technological solutions, there is not a unique technology that can fit all needs [10]. Since our main purpose is to develop tools that are either wearable by infants and suitable to be used in a minimally structured environment, the highest priority is given to technologies which are unobtrusive and ecological. For these reasons, the most appealing technology is represented by the sourceless inertial/magnetic sensing for orientation tracking as well as for linear accelerations. Such a technology is based on accelerometers and magnetometers used to sense respectively the gravitational and the geomagnetic field, as well as on gyroscopes used to enhance performance at higher frequencies. A complementary filter approach is used to derive $3 \mathrm{D}$ orientation from inertial and magnetic measurements [14]. Although there are several commercial systems available (InterSense, Xsens, etc.), these ones are not suitable for newborn movement analysis mainly because of their size and weight, so a custom solution is required.

The full scale range of a sensor, given the full scale range of a set of available sensors, is selected as the minimum one containing the whole signal excursion. A smaller range may in fact lead to saturation when certain operative condition occurs, while a larger range would reduce sensitivity.
In order to estimate a proper scale range preliminary quantitative experiments have been conducted. A threecamera $(500 \mathrm{~Hz})$ motion capture system was used (Qualysis Motion Capture Systems Inc., SWE) at University of Edinburgh to obtain position-time data from both arms of infants and evaluate frequency and dynamic content of their spontaneous movements. Three reflective non linear markers were placed on the head, respectively on the right, left and top head side, and two on the hands, right and left. In Fig. 1A data on one week old male infant are shown. Accelerations with respect to a fixed reference frame were calculated from position data of wrist markers via a double time derivative (Fig. 1.B).

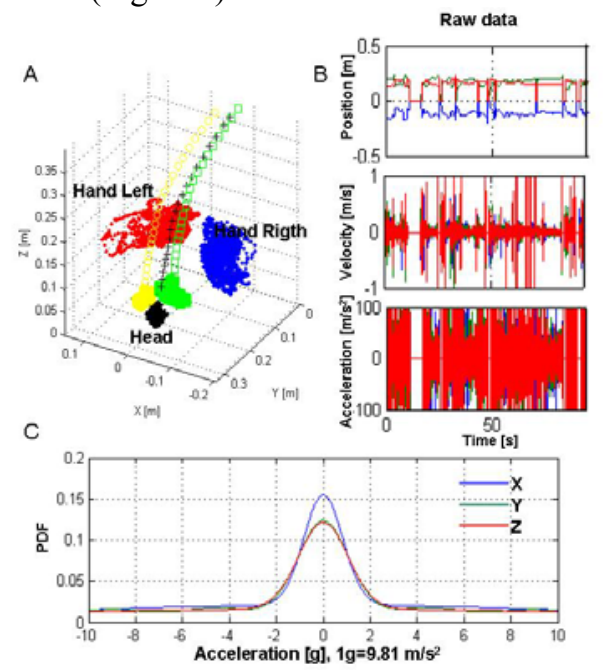

Fig. 1 Preliminary kinematics analysis on one week old male infant: (A) raw position data; (B) acceleration and velocity reconstructed by time derivative from raw position data; (C) Probability Distribution Function (PDF) of the acceleration.

Velocity and acceleration are very noisy due to numerical estimation and spikes generated when the markers are out of sight. Probability Density Function (PDF) has been calculated $^{1}$ to split noise from signal in order to define the dynamic range for the accelerometer sensors. It shows that accelerations are included in the $\pm 2 g$ range (Fig. 1.C).

As for the magnetometers, since only the geomagnetic field (about 0.6 gauss) needs to be measured, the full measurement range should be in the order of \pm 1 Gauss.

Saturation of gyroscopes would result in a problematic loss of tracking and therefore, given several commercially available gyroscopes with full scale ranging from \pm 150 $\mathrm{deg} / \mathrm{s}$ to $\pm 1200 \mathrm{deg} / \mathrm{s}$, the maximum scale range $( \pm 1200$ $\mathrm{deg} / \mathrm{s}$ ) was selected.

\section{Bandwidth and $A / D$ conversion}

The bandwidth of the signal was also estimated in order to select an appropriate filter and to improve the Signal to Noise Ratio (SNR). Using MATLAB spectrogram function, the Power Spectral Density was computed. Raw position

\footnotetext{
${ }^{1}$ Computed via ksdensity() MATLAB function.
} 
data have been segmented in vectors of 256 samples and the normalize short time Fourier transform has been calculated for each vector. Results in Fig. 2 show that the signal can be considered band limited at $50 \mathrm{~Hz}$. Traditional marker based motion capture systems have high frequency sampling cameras $(100-500 \mathrm{~Hz})$ which are used to film experimental sessions from different view points.

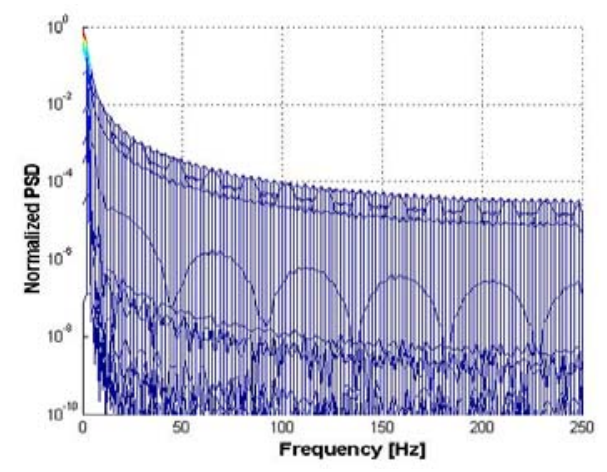

Fig. 2 Power spectral density of the accelerations

In line with such specification a sampling frequency of 250 $\mathrm{Hz}$ has been chosen. This frequency is between the sampling frequency of other magneto-inertial commercially available systems and the frequency of the optical devices. This value is not strictly necessary because a $100 \mathrm{~Hz}$ sampling frequency should be enough according to Shannon theorem, anyway it has been chosen for two main reasons: $i$ ) it allows an easier interfacing with other marker based motion capture devices by means of synchronization; ii) it allows to reduce quantization error via over sampling techniques.

\section{Design AND FABRication of WAMS Device}

In this section design and assembling of the first prototype of WAMS device is discussed according to specifications detailed above.

\section{A. Mechanical design}

A first rigid structure of $40 \times 30 \mathrm{~mm}$ has been designed and fabricated (Fig. 3.A) by a 3D fast prototyping printer from 3D ZCorporation, Inc. Such a mechanical solution is made by two parts:

1. a support structure designed to bind up infant's limbs;

2. a covering shell devoted to improve the infant's safety level and to avoid any possible contact between electric components and the infant body.

Although this structure fits the mechanical constraints detailed above, a big problem is given by its weight and rigidness: the support structure alone, without covering shell and electric components, is $25 \mathrm{~g}$ in weight; the rigid material does not allow for a comfortable wearing by subject with different anatomical structures.

For improving the anatomical adaptability and, at the same time, to reduce the total weight of the device, the structure has been realized by means of a silicon rubber material which does not need any mounting screws potentially dangerous for the child. The chosen rubber is the Silplay RTV 530 made by Prochima: it is an edible silicon used in the food industry. The structure has been realized in two steps: in the first one, rubber was dripped in a mold to form the flexible support structure; in the second one, the Velcro straps and electronic board were put into the mold on four square supports (Fig. 3.B) and a new rubber casting was made so that electronic and Velcro straps were embedded into the final structure. The total weight of the silicon support structure (Fig. 3.C) and electronic board is about $20 \mathrm{~g}$ i.e. 10 grams lighter than state of the art magnetoinertial device commercially available and about $40 \%$ smaller.
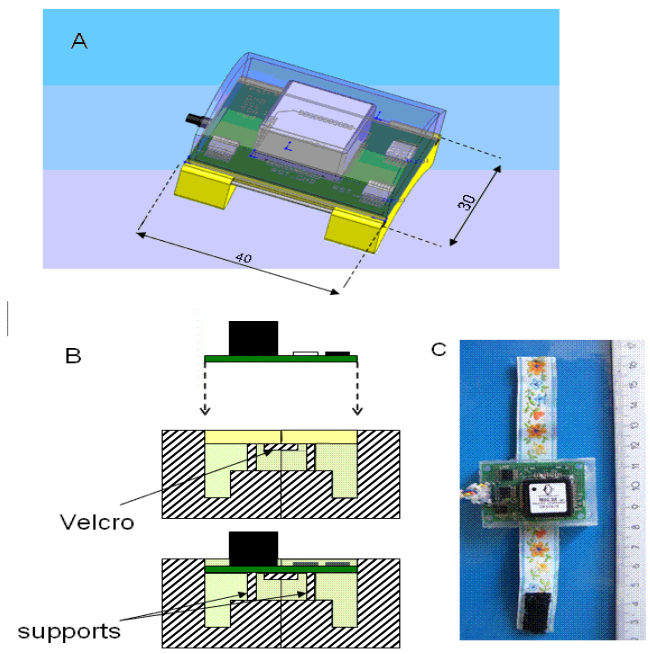

Fig. 3 Mechanical design: (A) First design; (B) ; Fabrication steps; (C) Final result: the silicon support structure embedding electronic board and Velcro straps.

\section{B. Kinematics Sensing Unit}

The inertial/magnetic technology requires accelerometers, gyroscopes and magnetometers: although there are several highly miniaturized components available off the shelf, a mircrofabricated device integrating all the required sensing capability has been chosen. There are two main reasons for such a choice:

1. a more efficient packaging;

2. a more reliable axis orientation. Not orthogonal sensible axes directly translate in errors during orientation tracking.

A preliminary analysis of commercial magneto-inertial sensors at present available has highlighted a triaxial magnetometer, accelerometer and gyroscope analog sensor 
from Memsense (MAG02-1200S050) which matches technical requirements previously detailed (see Table III). TABLE III

TECHNICAL SPECIFICATIONS OF MAG02-1200S050

\begin{tabular}{ll}
\hline \hline Technical characteristics & Value \\
\hline Accelerometers range $\left[\mathrm{m} / \mathrm{s}^{\wedge} 2\right]$ & $\pm 2 \mathrm{~g}$ \\
Magnetometers range [Gauss] & \pm 1.9 \\
Gyroscopes range [ $/ \mathrm{s}]$ & 1200 \\
Size $[\mathrm{mm}]$ & $17 \times 18 \times 10$ \\
Bandwidth $[\mathrm{Hz}]$ & 50 \\
Weight $[\mathrm{g}]$ & 5 \\
\hline \hline
\end{tabular}

For this reason it has been chosen for the first WAMS prototype. It only requires accelerometers and magnetometers signals to be buffered. An in-field calibration like the one in [15] can be used.

\section{A/D Conversion, Data Acquisition and Communication Protocol}

Data can be digitally converted via several MAX1238 (lowpower, 12-channaels, 12 bits AD Converters from MaximDallas). After conversion, data are sent to a remote master station via $\mathrm{I} 2 \mathrm{C}$ standard protocol. In this station a microcontroller PIC16F877A collects data from every MAX1238 (each identified by a unique address) and retransmits them via RS232 to a PC where they are stored (Fig. 4.A).

The communication with the four devices has been made by messages which are built according to a standard structure (Fig. 4.B). The message has two parts; one with a set of control communication fields (PREAMBLE, ID, and CHECKSUM) and one made by time stamps and sensors data bytes. Using this configuration the message has a total of 23 bytes. For the chosen sampling rate of $250 \mathrm{~Hz}$ this message structure results in a bit rate of $230 \mathrm{kbps}$ when 4 WAMSs devices are used in parallel. Such a high bit rate requires to split the communication data stream on two serial ports.

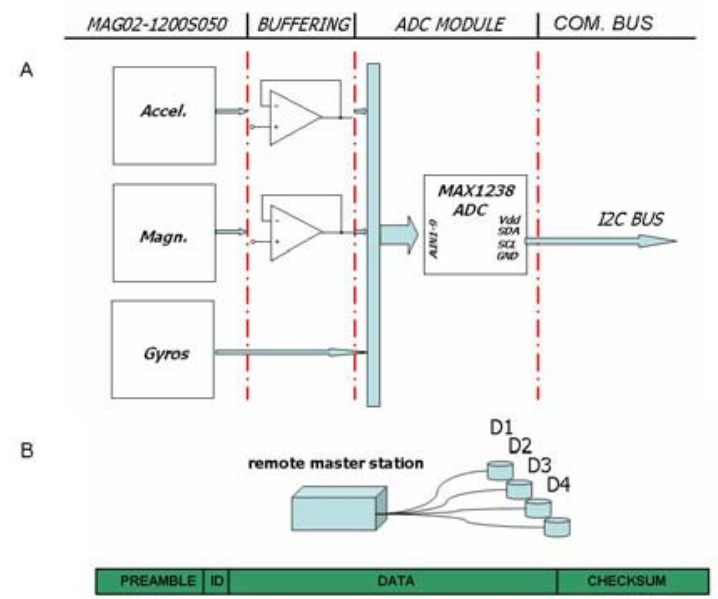

Fig. 4 A/D conversion and communication protocol: (A) logic diagram of A/D conversion; (B) communication protocol
A first Printed Circuit Board (PCB) has been designed and produced (Fig. 5A). Its final dimensions fit mechanical constraints detailed in the functional and technical specifications section. Power supply comes from a battery placed in the remote master station and a four poles cable is used to take (digital) signals out and power-ground in: this is done to preserve the child's safety and to reduce the weight of the final device.

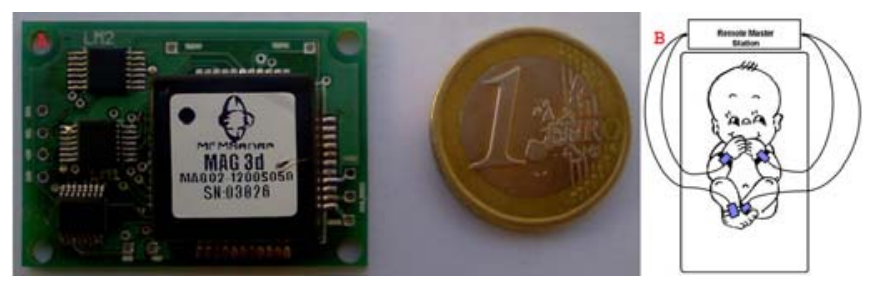

Fig. 5 A) First prototype; B) Typical experimental set-up

The wired communication is not extremely troublesome, since in a typical experimental scenario (Fig.5.B), the infant is in a cradle and cannot walk yet.

\section{Synchronization device}

The proposed magneto-inertial system should be used as a standalone application or with other motion capture systems (in particular optical ones like Qualisys) in order to reduce their complexity level, e.g. the number of necessary cameras. In this section an easy and general device to synchronize magneto-inertial data stream with the one coming from other optical motion capture systems is presented. Although several of these devices have some kind of synchronization ports, an home made solution has been chosen in order to have a more general and flexible device. For this reason a serial monitor has been designed. Such a device should monitor serial data coming from WAMS, and provide a synchronization signal based on the timer of the read message (for example switching on and off a control led). This signal will be used to mark each video frame gathered by the used optical motion capture system and to guarantee the alignment between the two data streams. In the first prototype, a predefined sequence of three coloured leds has been defined. Each step of this sequence is executed at regular time intervals (about 36,72 s) and a contemporary acoustic signal is generated at a predefined frequency (this allows the synchronization with an audio stream too). When the time field of the WAMS data stream saturates the led sequence starts again and so on.

\section{PRELIMINARy Data}

A preliminary test to verify data acquired by means of WAMS device has been performed. WAMS has been fixed into a rigid box together with a commercial magneto-inertial measurement unit, MTx from Xsens technologies . Sensible axes have been manually aligned. Two slow rotations, 
respectively around $\mathrm{x}$ and $\mathrm{z}$ axis, have been performed and accelerations collected by means of the two devices have been compared. The slowness of the movement allows us to consider only the gravitational acceleration known. Data acquired during trials are consistent with orientation of the mobile reference frame with respect to the gravitational field. Moreover, despite the manual alignment and the different positions of the two devices, data gathered by WAMS fit data gathered by MTx device (see Fig.6.A).
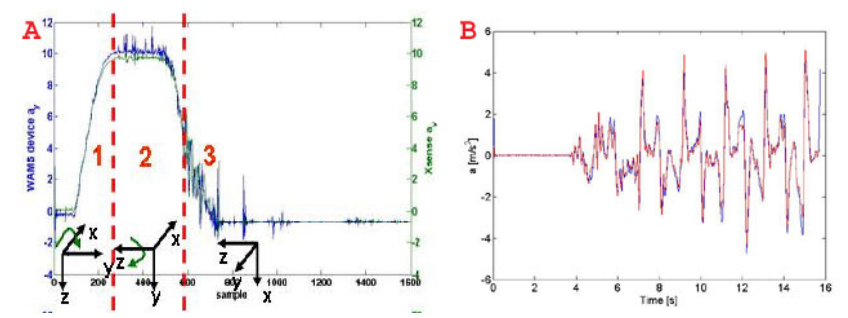

Fig. 6 A) Measured acceleration with respect to $y$ axis. Orientation of the mobile system before (1) and after (2), (3) the rotation movements is shown; B) Acceleration comparison: the red trace is the acceleration measured by the WAMS, the blue trace is the acceleration calculated from Qualysis position data

The main goal of WAMS device was to solve and improve respectively occlusion and portability issues related to some movement analysis systems like optical ones. When these systems are used to study limbs movements of newborns usually a fixed axis aligned to the head-foot axis is considered. The reason for this simplification is related to the fact that newborns usually tend to shake their limbs up and down along this direction.

According to such simplification a first test using WAMS device and Qualysis motion capture system has been executed at Perception in Action Laboratory at Edinburgh University. A marker has been placed on the WAMS device and a common fixed reference frame has been defined for the two measurement systems. An up and down movement along one of the horizontal axes of the fixed reference frame (the $\mathrm{y}$ one) has been executed mimicking newborns' movements. Acceleration measured by WAMS and the ones calculated by means of a double derivation of position data measured by Qualysis have been compared along y axis of the fixed reference frame (Fig. 6.B). The mean error between the two traces is $0.0023 \mathrm{~m} / \mathrm{s}^{\wedge} 2$ which is comparable with the electrical noise of the accelerometer sensors in the WAMS device.

The second test performed was related to the possibility of reconstructing position and velocity data along the y-axis in case of marker occlusion. Velocity and acceleration can be calculated respectively as the first and second derivative of the position data. Let $\mathrm{y}[\mathrm{n}], \mathrm{n} \in[1, \mathrm{~N}]$ be the position data measured by Qualysis at frequency fs and $\Delta \mathrm{T}=\mathrm{fs}^{-1}$, the time interval between each sample. Velocity and acceleration calculated by position data are:

$$
\begin{aligned}
& \dot{y}[i]=\frac{y[i+1]-y[i]}{\Delta T}, \mathrm{i} \in[1, \mathrm{~N}-1] \\
& \ddot{y}[j]=\frac{\dot{y}[j+1]-\dot{y}[j]}{\Delta T}, \mathrm{j} \in[1, \mathrm{~N}-2]
\end{aligned}
$$

Supposing a marker occlusion between sample $\mathrm{n}_{0}$ and $\mathrm{n}_{0}+\mathrm{K}$, velocity should be set to zero up to sample $n_{0}+K$ where it should have a spike:

$$
\dot{y}\left[n_{0}+K\right]=\frac{\dot{y}\left[n_{0}+K+1\right]-\dot{y}\left[n_{0}+K\right]}{\Delta T}=\frac{\dot{y}\left[n_{0}+K+1\right]-0}{\Delta T}
$$

Theoretically, it is possible to reconstruct velocity and position data using acceleration $\mathrm{a}[\mathrm{n}], \mathrm{n} \in[1, \mathrm{~N}]$ measured by a different measurement system by means of a double integration using suitable initial conditions. Unfortunately, using magneto-inertial technologies, acceleration signal $\left(\operatorname{acc}_{\text {out }}[\mathrm{n}]\right)$ is affected by a low frequency noise $a_{n}[n]$ :

$$
\operatorname{acc}_{\text {out }}[n]=a[n]+a_{n}[n]
$$

When it is integrated, the estimated velocity in the interval $\left[\mathrm{n}_{0}-1, \mathrm{n}_{0}+\mathrm{K}\right]$ is:

$$
\begin{aligned}
& \tilde{\dot{y}}[h]=\sum_{h=n_{0}}^{n_{0}+K} \frac{a[h]+a[h+1]}{2} \Delta T+\ldots \\
& \ldots+\sum_{h=n_{0}}^{n_{0}+K} \frac{a_{n}[h]+a_{n}[h+1]}{2} \Delta T+\ldots \\
& \ldots+\dot{y}\left[n_{0}-2\right]+\ddot{y}\left[n_{0}-2\right] \Delta T
\end{aligned}
$$

where:

- $\sum_{h=n_{0}}^{n_{0}+K} \frac{a[h]+a[h+1]}{2} \Delta T$ is equal to the real velocity $\dot{y}$;

- $\quad \sum_{h=n_{0}}^{n_{0}+K} \frac{a_{n}[h]+a_{n}[h+1]}{2} \Delta T$ is the drift error e[h] due to noise, i.e the difference between the real and the estimated velocity $e[h]=\tilde{\dot{y}}[h]-\dot{y}[h], \mathrm{h} \in\left[\mathrm{n}_{0}\right.$, $\left.\mathrm{n}_{0}+\mathrm{K}\right]$

- $\quad \dot{y}\left[n_{0}-2\right]+\ddot{y}\left[n_{0}-2\right] \Delta T$ is the initial condition.

Assuming a linear drift function, i.e. a constant low frequency noise $\left(\left(a_{n}[h]+a_{n}[h+1]\right) / 2=a_{n}, \forall h\right)$, the drift error at sample $\mathrm{n}_{0}+\mathrm{K}$ will be $\mathrm{e}\left[\mathrm{n}_{0}+\mathrm{K}\right]=\mathrm{Ka}_{\mathrm{n}} \Delta \mathrm{T}$ from which electrical low noise will be:

$$
a_{n}=\frac{e\left[n_{0}+K\right]}{K \Delta T}
$$

Knowing the real velocity at sample $\mathrm{n}_{0}+\mathrm{K}+1$, the drift error can be estimated as:

$$
\begin{aligned}
& e\left[n_{0}+K\right]=\tilde{y}\left[n_{0}+K\right]-\left(\dot{y}\left[n_{0}+K\right]+\ldots\right. \\
& \left.-\ldots \ddot{y}\left[n_{0}+K\right] \Delta T\right)
\end{aligned}
$$

Substituting (6) in (5) it is possible to have an assessment of electrical low frequency noise which can be used to adjust estimated velocity:

$$
\hat{\dot{y}}[h]=\widetilde{\dot{y}}[h]-\left(h-n_{0}\right) a_{n} \Delta T, h \in\left[n_{0}, n_{0}+K\right]
$$


Where $\hat{y}[h]$ is the adjusted estimated velocity. A marker occlusion problem has been simulated filling portions of position data (between sample 1730 and sample 1830) with a sequence of 100 samples set to zeros (Fig. 7)

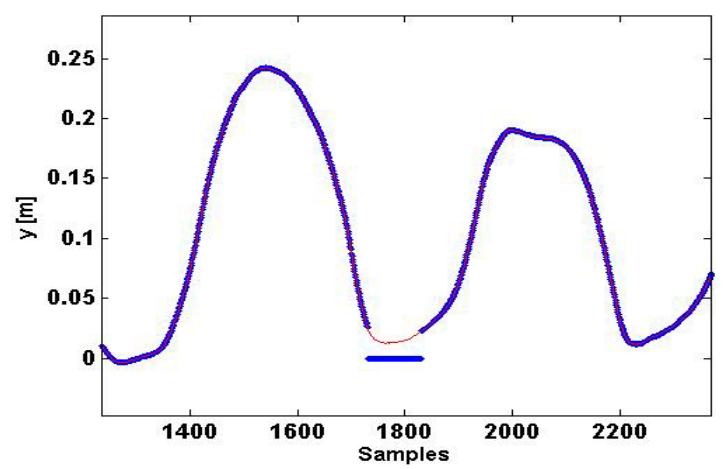

Fig. 7 Simulated marker occlusion; red trace, real data; blue trace, simulated data

In (Fig. 8), velocity has been calculated from acceleration according to methodology discussed above: the red trace is the real velocity, the blue dotted trace is the estimated velocity $\widetilde{\dot{y}}$ with drift error and the dashed blue trace is the adjusted estimated velocity $\hat{\dot{y}}_{\dot{\hat{j}}}$ The same process can be used to estimate position from $\dot{y}(t)$ ( Fig. 8.B).
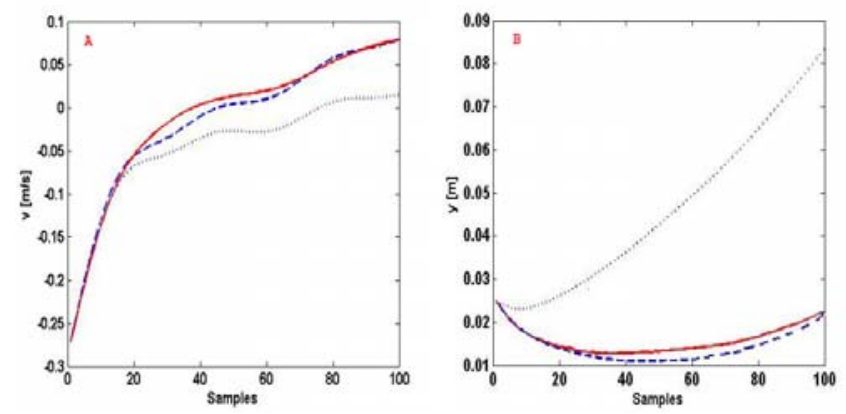

Fig. 8 Velocity (A) and position (B) calculated from accelerometers data

\section{CONCLUSION AND FUtURE WORK}

In this work a mechatronic wearable device for ecological movement analysis of infants aged between 1 week and 12 months called WAMS (Wrist and Ankle Movement Sensor) has been designed and developed. It has been devised to be used as a stand-alone solution for movement analysis as well as part of a pre-existing setup by means of synchronization.

Preliminary data on spontaneous movements of one week old male infant have been gathered by means of an optical marker-based system. Such data have been used to set the band of the signal and to guide the choice of the sensing unit. The design of the mechanical support as well as the communication protocol and synchronization system have been discussed.

A preliminary comparison between WAMS device and a commercially available magneto-inertial system has been carried out.

Finally WAMS device and an optical motion capture system has been compared: in particular the possibility to have the same information about acceleration along a principal movement axis has been verified. A data losing has been simulated and the possibility to reconstruct data trace from the signal coming from WAMS has been tested. Preliminary results seem to be promising.

A second version of WAMS device is under development. Its final weight should be under $10 \mathrm{~g}$ at all and its size about $2.5 \times 2.5 \mathrm{~mm}$. The performance of data reconstruction will be characterized, in particular it will be analyzed the error of reconstruction for different time interval.

\section{REFERENCES}

[1] J. H. Martin, D. Engber, Z. Meng, "Effect of forelimb use on postnatal development of the forelimb motor representation in primary motor cortex of the cat.", Journal of Neurophysiology, Vol. 93, pp 28222831, Mar. 2005.

[2] J. J. Lockmann, "A Perception-Action Perspective on Tool Use Development”, Child Development, Vol. 71, No. 1, pp. 137-144, Jan/Feb 2000

[3] C. von Hofsten, L. Ronquist "The Structuring of Neonatal Arm Movements", Child Developments, Vol. 64, pp. 1046-1057, 1993.

[4] P. Teitelbaum, O. Teitelbaum, J. Nye, J. Fryman, R. G. Maurer, "Movement analysis in infancy may be useful for early diagnosis of autism”, Proc Natl Acad Sci USA 95: 13982-13987.

[5] O. Teitelbaum, T. Benton, P.K. Shah, A. Prince, J. L. Kelly, P. Teitelbaum "Eshkol-Wachman movement notation in diagnosis: The early detection of Asperger's syndrome", Proc Natl Acad Sci, vol. 101(32) , pp. 11909-11914, Aug. 2004. Available: http://www.pnas.org/cgi/reprint/0403919101v1.pdf

[6] C. Trevarthen, S. Daniel, "Disorganized rhythm and synchrony: Early signs of autism and Rett syndrome", Brain \& Development, Vol.27, pp. S25-S34, Nov. 2005.

[7] A. N. Bhat, J. C. Galloway. "Toy-oriented changes during early harm movements: Hand Kinematics", Infant Behaviour and Development, Vol. 29, pp. 358-372, Jan. 2006.

[8] A. N. Bhat, J. H. Heathcock, J. C. Galloway. "Toy-oriented changes in hand and joint kinematics during the emergence of purposeful reaching.", Infant Behaviour and Development, Vol. 28, pp. 445-465, 2005.

[9] S. Ohgi, K.K. Loo, S. Morita, C. Mizuike, "A Dynamical Systems Analysis of Spontaneus Movements in Newborn infants", Journal of Motor Behaviour, Vol. 37(3), pp. 203-214, May 2007

[10] G. Welch and E. Foxlin, "Motion Tracking: No Silver Bullet, but a Respectable Arsenal", IEEE Computer Graphics and Applications, Vol. 22, No. 6, pp. 24-38, 2002.

[11] D. Campolo, E. S. Maini, F. Patanè, C. Laschi, P. Dario, F. Keller, E. Guglielmelli "Design of a Sensorized Ball for Ecological Behavioral Analysis of Infants", ICRA 2007

[12] Highway Safety Research Institute the university of Michigan Ann Arbor, Michigan 48109, " Antropometry of infants, children and youths to age 18 for product safety design", May 311978

[13] Schneider, K. and Zernicke, R. F. Mass, center of mass, and moment of inertia estimates for infant limb segments. J. Biomech. 25: 145148, 1992.

[14] D. Campolo, F. Keller, E. Guglielmelli, "Inertial/Magnetic Sensors Based Orientation Tracking on the Group of Rigid Body Rotations with Application to Wearable Devices", IEEE/RSJ Int. Conf. on Intelligent Robots and Systems (IROS), Beijing, P.R. China, October 9-14, 2006.

[15] D. Campolo, M. Fabris, G. Cavallo, D. Accoto, F. Keller, E. Guglielmelli, "A Novel Procedure for In-field Calibration of Sourceless Inertial/Magnetic Orientation Tracking Wearable Devices", the first IEEE / RAS-EMBS Intl Conf. on Biomedical Robotics and Biomechatronics (BIOROB), Pisa, Italy, February 20-22, 2006. 